\title{
Family health history reporting is sensitive to small changes in wording
}

\author{
Liam S. Conway-Pearson, BA ${ }^{1}$, Kurt D. Christensen, $\mathrm{PhD}^{2,3}$, Sarah K. Savage, MS ${ }^{4}$, \\ Noelle L. Huntington, PhD ${ }^{5,6}$, Elissa R. Weitzman, $\mathrm{ScD}$, MSc ${ }^{6,7,8}$, Sonja I. Ziniel, $\mathrm{PhD}^{6,7,9}$, \\ Phoebe Bacon, BA ${ }^{10}$, Cara N. Cacioppo, BA ${ }^{4}$, Robert C. Green, MD, MPH ${ }^{2,3,11}$ \\ and Ingrid $\mathrm{A}$. Holm, MD, MPH $\mathrm{MP}^{4,6,12}$
}

Purpose: Family health history is often collected through singleitem queries that ask patients whether their family members are affected by certain conditions. The specific wording of these queries may influence what individuals report.

Methods: Parents of Boston Children's Hospital patients were invited to participate in a Web-based survey about the return of individual genomic research results regarding their children. Participants reported whether 11 types of medical conditions affected them or their family. Randomization determined whether participants were specifically instructed to consider their extended family.

Results: Family health history was reported by 2,901 participants. Those asked to consider their extended family were more likely

\section{INTRODUCTION}

Family health history has well-established utility for diagnosing disease, optimizing treatment and prevention decisions, and motivating lifestyle changes to prevent disease. ${ }^{1}$ Despite its clear utility, family history is often collected quickly, using short instruments. A "full" family history includes three generations of relatives, health problems with age of onset for each family member, and age of each relative at death with cause.,3 Collecting and reviewing this information can take up to $30 \mathrm{~min}$ and strain routine health appointments, which average $15 \mathrm{~min}$ in duration. ${ }^{4}$ In addition, methods for collecting family history information vary greatly from clinic to clinic., ${ }^{5,6}$ The result is that clinicians and their staff are collecting crucial family history information in ways that not only are abbreviated but also may yield disparate results.?

The importance of a positive history of disease depends on the degree of relatedness of affected family members to the patient. The implications of a positive family history of disease on an individual's risk for disease are typically stronger when affected family members are more closely related. Risks for type 1 diabetes, for instance, are nearly 8.7 times greater when an individual has an affected first-degree relative, but only 1.7 to report a positive family history for 8 of 11 medical conditions. The largest differences were observed for cancer (65.1 vs. $45.7 \%$; $P<0.001$ ), cardiovascular conditions (72.5 vs. 56.0\%; $P<0.001$ ), and endocrine/hormonal conditions (50.9 vs. $36.7 \%$; $P<0.001$ ).

Conclusions: Small alterations to the way family health history queries are worded can substantially change patient responses. Clinicians and researchers need to be sensitive about patients' tendencies to omit extended family from health history reporting unless specifically asked to consider them.

Genet Med advance online publication 5 May 2016

Key Words: clinical practice; family history; research methods

times greater when an individual has an affected third-degree relative. ${ }^{8}$ At the same time, ignoring more distant relatives can diminish the utility of family history information. Pathogenic genetic variants may not be expressed or noticed in immediate relatives due to incomplete penetrance, variable expressivity, or recessive inheritance patterns, but still may be disease-causing in extended family members. Yet, a common strategy for collecting family health history is to ask patients simply to report whether they have a family history of certain conditions, leaving it up to patients to determine which relatives are relevant. ${ }^{79,10}$ In this short report, we report how explicitly asking patients to consider their extended family members during brief family history queries affects their likelihood of reporting each of 11 conditions.

\section{MATERIALS AND METHODS}

\section{Overview, randomization, and measures}

We present secondary findings from a Web-based survey. Detailed methods and primary findings are reported elsewhere. ${ }^{11,12}$ Briefly, parents of Boston Children's Hospital (BCH) patients were invited to participate in a study about receiving genomic research results about their children. The study was

\footnotetext{
${ }^{1}$ Northwestern University, Evanston, Illinois, USA; ${ }^{2}$ Department of Medicine, Brigham and Women's Hospital, Boston, Massachusetts, USA; ${ }^{3}$ Department of Medicine, Harvard Medical School, Boston, Massachusetts, USA; ${ }^{4}$ Division of Genetics and Genomics, Boston Children's Hospital, Boston, Massachusetts, USA; ${ }^{5}$ Division of Developmental Medicine, Boston Children's Hospital, Boston, Massachusetts, USA; ${ }^{6}$ Department of Pediatrics, Harvard Medical School, Boston, Massachusetts, USA; ${ }^{7}$ Division of Adolescent/Young Adult Medicine, Boston Children's Hospital, Boston, Massachusetts, USA; ${ }^{8}$ Children's Hospital Informatics Program at the Harvard-MIT Division of Health Sciences and Technology, Boston Children's Hospital, Boston, Massachusetts, USA; ${ }^{9}$ Center for Patient Safety and Quality Research, Boston Children's Hospital, Boston, Massachusetts, USA; ${ }^{10}$ Johns Hopkins University School of Medicine, Baltimore, Maryland, USA; ${ }^{11}$ Partners Personalized Medicine, Boston, Massachusetts, USA; ${ }^{12}$ Manton Center for Orphan Disease Research, Boston Children’s Hospital, Boston, Massachusetts, USA. Correspondence: Kurt D. Christensen (kchristensen@genetics.med.harvard.edu)
} 
designed to explore whether satisfaction with enrollment in a hypothetical biobank was associated with having an ability to designate preferences regarding what results to receive. Using a computer algorithm, parents were randomized to one of four hypothetical biobanks to address primary aims of the research, including assessment of patient satisfaction with different approaches for returning genetic research results. The four arms were as follows: (i) group 1a (15\%), which received no results; (ii) group $1 \mathrm{~b}(15 \%)$, which received all results; (iii) group $2(30 \%)$, which gave parents a choice of receiving all or no results; and (iv) group 3 (40\%), which used a preferencesetting tool to choose categories of results to disclose (groups 2 and 3 were proportionally larger to power within-group subanalyses about how specific preferences were associated with satisfaction).

Enrolled participants first watched a 5-min educational video about genetics and health and then were asked to report demographic information. Next, they reviewed a checklist of 11 classes of diseases (e.g., "cancer," "kidney or urinary condition"). All participants were supposed to receive identical surveys for the collection of family history and other background information. However, because of a programming error, participants in three of the four randomization arms were instructed, "Please check any medical conditions that affect you or your family"; participants in the fourth arm received a slightly modified statement: "Please

Table 1 Participant characteristics by randomization status

\begin{tabular}{|c|c|c|c|}
\hline $\begin{array}{l}\text { Characteristic: } \boldsymbol{n}(\%) \\
\text { unless noted }\end{array}$ & $\begin{array}{c}\text { Standard query } \\
(n=1,780)\end{array}$ & $\begin{array}{l}\text { Asked for extended } \\
\text { family }(n=1,137)\end{array}$ & $P$ \\
\hline Age (SD) & $42.8(7.4)$ & $43.0(7.5)$ & 0.623 \\
\hline Female & $1,627(91.4 \%)$ & $1,047(92.1 \%)$ & 0.517 \\
\hline \multicolumn{4}{|l|}{ Race $^{\mathrm{a}}$} \\
\hline Caucasian & $1,623(91.2 \%)$ & $1,014(89.2 \%)$ & 0.074 \\
\hline African American & $47(2.6 \%)$ & $21(1.8 \%)$ & 0.166 \\
\hline Asian & $68(3.8 \%)$ & $41(3.6 \%)$ & 0.766 \\
\hline Other & $30(1.7 \%)$ & $24(2.1 \%)$ & 0.406 \\
\hline \multicolumn{4}{|l|}{ Ethnicity } \\
\hline Hispanic & $123(6.9 \%)$ & $61(5.4 \%)$ & 0.094 \\
\hline Education & & & 0.289 \\
\hline $\begin{array}{l}\text { High school graduate } \\
\text { or less }\end{array}$ & $304(17.4 \%)$ & $175(15.8 \%)$ & \\
\hline $\begin{array}{l}\text { 2-year or 4-year } \\
\text { college graduate }\end{array}$ & $663(37.9 \%)$ & $421(37.9 \%)$ & \\
\hline $\begin{array}{l}\text { More than 4-year } \\
\text { college degree }\end{array}$ & $781(44.7 \%)$ & $514(46.3 \%)$ & \\
\hline Not provided & $32(1.8 \%)$ & $27(2.4 \%)$ & 0.280 \\
\hline Works in healthcare & $427(24.0 \%)$ & $258(22.7 \%)$ & 0.420 \\
\hline $\begin{array}{l}\text { Prior participation in } \\
\text { research }\end{array}$ & $681(38.3 \%)$ & $401(35.3 \%)$ & 0.103 \\
\hline $\begin{array}{l}\text { Prior genetic testing } \\
\text { experience }\end{array}$ & $736(41.3 \%)$ & $452(39.8 \%)$ & 0.393 \\
\hline $\begin{array}{l}\text { Child diagnosed with } \\
\text { genetic disorder }\end{array}$ & $414(23.3 \%)$ & $261(23.0 \%)$ & 0.850 \\
\hline
\end{tabular}

aparticipants could endorse more than one race. check any medical conditions that affect you or your family (including your extended family)" (emphasis added). We used REDCap software to administer the survey. ${ }^{13}$ The protocol was approved by the BCH Institutional Review Board (IRB-P00006896), including implied consent from participants through survey completion.

\section{Study population}

Eligible individuals, per pre-established criteria, were parents or guardians of children who had received care at $\mathrm{BCH}$ within 2 years of the study and whose e-mail addresses were in their medical records. Inclusion criteria were living in the United States, being 18 years of age or older, and having at least one child under 18 years of age at the time of participation. Parents were excluded if either the parent or their child was currently or previously enrolled in the Gene Partnership, a $\mathrm{BCH}$ pediatric biobank that offers the return of research results.

Potential participants were mailed a prenotification letter that explained the background and process of the study and included the e-mail address we planned to use for recruitment. The letter also indicated that participants who completed the survey would be entered into a raffle for one $\$ 100$ Visa gift card for every 100 completed surveys. Nine days after the prenotification letter was mailed, the survey invitation e-mail was sent to parents. During the 3 months that the survey was open, participants were sent a maximum of four reminder e-mails over the span of 5 weeks

\section{Data analysis}

We used R (version 3.2.2; http://www.R-project.org) to analyze the data. To compare participants who discontinued the survey before reporting personal and family history information and participants who reported that data, we used $t$-tests, chi-squared tests, Fisher exact tests, and Wilcoxon rank sum tests. We used $t$-tests and multiple linear regression to compare the total number of conditions reported by randomizationarm participants after examination for normal distributions using histograms; we used chi-squared tests to compare randomization arms regarding specific conditions. We used adapted McNemar tests ${ }^{14}$ to determine whether the impact of the extended-family prompt differed between conditions. Analyses excluded participants who dropped out of the study prior to the query for personal and family history information. Data from the three randomization-arm participants who were not given instructions to consider extended family were pooled if there were no differences in study procedures prior to reporting personal and family history and no differences in demographics. The sample size was established to test primary study hypotheses about differences in satisfaction, ${ }^{11}$ but post hoc analyses suggested $99 \%$ power to detect small differences (i.e., Cohen's $d=0.2$ for $t$-tests, 0.1 for chi-squared tests) by randomization status in analyses comparing randomization arms at a significance level of $P=0.01$. All statistical tests were two-sided, with significance set at $P=0.01$ to account for the large number of analyses. 


\section{Code availability}

The study protocol, statistical code, and data set are available from Dr Holm (Ingrid.Holm@childrens.harvard.edu) upon request.

\section{RESULTS}

A total of 3,007 eligible parents started the survey, and 2,917 of them $(97.0 \%)$ provided family history information. Individuals were more likely to terminate the survey before providing family history information if they had been randomized to consider extended family than if they had not (4.1 vs. $2.3 \%$, respectively; $P=0.006$ ). Participants with no prior experience with genetic testing were also more likely to terminate the survey early compared to participants with genetic testing experience (3.7 vs. $1.7 \%$, respectively; $P=0.001$ ). No other differences in dropout were observed regarding demographic or experiential factors. The characteristics of participants who provided family history information are summarized in Table 1 . No differences were observed regarding demographic or experiential factors by randomization status.

Personal information and family history information are summarized in Table 2. For all but three conditions, participants who were prompted about extended family reported a higher prevalence of conditions $(P<0.01)$. On average, participants reported 4.4 conditions $(\mathrm{SD}=2.5)$ when they were prompted to consider their extended family and 3.4 conditions ( $\mathrm{SD}=2.3)$ when the prompt was omitted $(\Delta=1.0 ; 99 \%$ CI: 0.8 to $1.3 ; P<0.001)$. Analyses suggested that the impact of the extended-family prompt varied by condition. The strongest effects were observed for cancer, for which the impact of the prompt on the odds of reporting a positive family history was 2.8 times greater than the impact on the odds of reporting kidney or urinary conditions and 2.2 times greater than the impact on the odds of reporting lung conditions and developmental delays (both $P<0.001$ ). Multiple linear regression also showed that participants reported more conditions if they were female ( $\mathrm{B}=1.17 ; P<0.001)$, older $(\mathrm{B}=0.02 /$ year; $P<0.001)$, selfidentified as a race other than Caucasian $(B=0.76 ; P<0.001)$, less educated $(\mathrm{B}=0.43$ for college degree or less compared with more than college degree; $P<0.001)$, had previously participated in research $(B=0.39 ; P<0.001)$, or had previous genetic testing experience $(\mathrm{B}=0.45 ; P<0.001)$.

\section{DISCUSSION}

Here, we report one of the first studies to use a randomized design to understand the impact of asking patients to consider their extended family when reporting family history of disease. Although this was not the intention of the study, the error in programming gave us the unexpected opportunity to address this issue. Respondents were substantially more likely to report additional conditions when so prompted. Although best practices recommend much greater detail when collecting family history information, yes/no approaches are common in clinical and research settings when patients are not seeking care for a specific problem. Our findings may be particularly important in primary care, where a positive family history of disease may influence whether a physician recommends additional diagnostic workup.

Our data suggest that patients who are not prompted to consider extended family may tend to focus on first-degree relatives. For example, $37 \%$ of participants in our study who were not instructed to consider extended family reported a family history of endocrine or hormonal conditions. By comparison, 38\% of respondents to the 2009 National Health and Nutritional Examination Survey ${ }^{15}$ and 30\% of respondents to the 2005 Oregon Behavioral Risk Factor Surveillance System ${ }^{16}$ reported diabetes, the most common endocrine disorder, when instructions specifically asked respondents to consider firstdegree relatives. Whatever the reason, clinicians and researchers who are using brief instruments need to be sensitive to the large impact that small changes in wording can have. In particular, they should specify which family members they want patients to consider when they ask patients to report their family history, even when using brief items to collect this information. Decisions about whether and how to use brief items to collect family history information should also consider that the accuracy of reporting typically decreases as the degree of relatedness decreases. ${ }^{3}$

Table 2 Reported family history for 11 conditions by randomization status

\begin{tabular}{|c|c|c|c|c|}
\hline Condition & $\begin{array}{c}\text { Standard } \\
\text { query }(n=1,780)\end{array}$ & $\begin{array}{l}\text { Asked for extended } \\
\text { family }(n=1,137)\end{array}$ & $\begin{array}{l}\text { Difference } \\
(99 \% \mathrm{Cl})\end{array}$ & $P$ \\
\hline Cardiovascular condition & $997(56.0 \%)$ & $824(72.5 \%)$ & $16.3 \%(11.6$ to $21.7 \%)$ & $<0.001$ \\
\hline Arthritis & $564(31.7 \%)$ & $491(43.2 \%)$ & $11.4 \%(6.5$ to $17.1 \%)$ & $<0.001$ \\
\hline Mental health & $752(42.2 \%)$ & $596(52.4 \%)$ & $10.1 \%(4.6$ to $14.7 \%)$ & $<0.001$ \\
\hline Muscle or bone condition & $236(13.3 \%)$ & $214(18.8 \%)$ & $5.6 \%$ (3.0 to $17.3 \%)$ & $<0.001$ \\
\hline $\begin{array}{l}\text { Developmental delay, learning or behavioral } \\
\text { disorder, or intellectual disability }\end{array}$ & $571(32.1 \%)$ & $401(35.3 \%)$ & $3.0 \%(-2.3$ to $8.6 \%)$ & 0.100 \\
\hline Kidney or urinary condition & $196(11.0 \%)$ & $157(13.8 \%)$ & $2.8 \%(-1.6$ to $14.2 \%)$ & 0.023 \\
\hline Lung or respiratory condition & $328(18.4 \%)$ & $241(21.2 \%)$ & $2.7 \%(-2.4$ to $10.5 \%)$ & 0.078 \\
\hline
\end{tabular}


The impact of the extended-family prompt tended to be strongest for the most common conditions, including cancer and cardiovascular conditions. Participants reported family health history in a study about receiving research results about their children, and the prompt may have caused them to consider more distant family members or relatives of their partners or spouses. It is also possible that participants were aware of conditions affecting more distant relatives if multiple family members had been affected. Alternatively, participants may have simply assumed that they had more distant relatives affected by the more common conditions without knowing of specific instances.

Additional findings showing more reported conditions among women might be explained by their tendency to be caretakers of health information in families. ${ }^{17}$ The higher numbers we observed among older participants, individuals who did not self-identify as white, and less-educated participants are probably a reflection of well-documented health disparities, ${ }^{18}$ given that demographic factors tend to be shared within families (particularly close relatives). ${ }^{19}$ The prevalence of diabetes, for example, is almost $5 \%$ higher among black non-Hispanic adults than white non-Hispanic adults. ${ }^{20}$ Similarly, the prevalence of diabetes is at least 3\% lower among individuals with college degrees than individuals who are less educated..$^{20}$ The common use of family history as inclusion criteria for many studies may explain why stronger family histories of disease were observed among individuals with previous research experience. Findings about individuals with prior genetic testing experience were not surprising because strong family histories for disease suggest shared genetic risk factors that may be identified through testing.

Several limitations to our research exist. Our participants were predominantly well-educated white women. $\mathrm{BCH}$ does not systematically collect information about the parents of patients, so we cannot ascertain the representativeness of respondents. A substantial proportion of our participants worked in health care or had experience with research or genetic testing and therefore may have been more likely to include extended family members when thinking about family histories for disease.

In conclusion, even among a more informed population, our data highlight the strong effect of adding a few words to a brief family history query. Our findings will be of great value to clinicians and researchers as they strive to develop family history instruments that maximize both utility and ease of administration.

\section{ACKNOWLEDGMENTS}

This work was supported by NIH grants HG006615, HG006500, HD077671, and HG006993.

\section{DISCLOSURE}

R.C.G.'s research is supported by grants from the $\mathrm{NIH}$, the DOD, and Illumina. R.C.G. has received compensation for advisory services or speaking from Invitae, Prudential, Illumina, AIA, Helix, and Roche. The other authors declare no conflict of interest.

\section{REFERENCES}

1. Berg AO, Baird MA, Botkin JR, et al. National Institutes of Health State-ofthe-Science Conference Statement: Family History and Improving Health. Ann Intern Med 2009;151:872-877.

2. Bennett RL. The family medical history as a tool in preconception consultation. J Community Genet 2012;3:175-183.

3. Wattendorf DJ, Hadley DW. Family history: the three-generation pedigree. Am Fam Physician 2005;72:441-448.

4. Bruen BK, Ku L, LuX, Shin P. No evidence that primary care physicians offer less care to Medicaid, community health center, or uninsured patients. Health Aff (Millwood) 2013;32:1624-1630.

5. Wilson B, Qureshi N, Little J, et al. Clinical Utility of Cancer Family History Collection in Primary Care. Evid Rep Technol Assess 2009;179:1-94.

6. Tarini BA, McInerney JD. Family history in primary care pediatrics. Pediatrics 2013;132(suppl 3):S203-S210.

7. Wood ME, Stockdale A, Flynn BS. Interviews with primary care physicians regarding taking and interpreting the cancer family history. Fam Pract 2008;25:334-340.

8. Weires MB, Tausch B, Haug PJ, Edwards CQ, Wetter T, Cannon-Albright LA. Familiality of diabetes mellitus. Exp Clin Endocrinol Diabetes 2007;115: 634-640.

9. Daelemans S, Vandevoorde J, Vansintejan J, Borgermans L, Devroey D. The use of family history in primary health care: a qualitative study. Adv Prev Med 2013;2013:695763.

10. Martinez KA, Fagerlin A, Witteman HO, Holmberg C, Hawley ST. What matters to women when making decisions about breast cancer chemoprevention? Patient 2015; 9:149-159.

11. Holm IA, Iles BR, Ziniel SI, et al. Participant satisfaction with a preference-setting tool for the return of individual research results in pediatric genomic research. J Empir Res Hum Res Ethics 2015;10:414-426.

12. Bacon PL, Harris ED, Ziniel SI, et al. The development of a preference-setting model for the return of individual genomic research results. J Empir Res Hum Res Ethics 2015;10:107-120.

13. Harris PA, Taylor R, Thielke R, Payne J, Gonzalez N, Conde JG. Research electronic data capture (REDCap)-a metadata-driven methodology and workflow process for providing translational research informatics support. J Biomed Inform 2009;42:377-381.

14. Lienert GA, Marascuilo LA. Comparing treatment-induced changes for $k$ independent samples of paired observations. Biom J. 1980;22(8):763-777.

15. Centers for Disease Control and Prevention (CDC). National Center for Health Statistics (NCHS). National Health and Nutrition Examination Survey Data. 2010. http://wwwn.cdc.gov/nchs/nhanes/search/nhanes09_10.aspx. Accessed 12 January 2016.

16. Oregon Health Authority. 2009 BRFSS Results. 2016. https://public.health. oregon.gov/BirthDeathCertificates/Surveys/AdultBehaviorRisk/brfssresults/ Pages/brfsqu09.aspx. Accessed 12 January 2016.

17. Emslie C, Hunt K, Watt G. A chip off the old block? Lay understandings of inheritance among men and women in mid-life. Public Underst Sci 2003;12: 47-65.

18. Meyer PA, Penman-Aguilar A, Campbell VA, Graffunder C, O'Connor AE, Yoon PW. Conclusion and future directions: CDC Health Disparities and Inequalities Report-United States, 2013. MMWR Surveill Summ 2013;62(suppl 3):184-186.

19. Coleman JS. Social capital in the creation of human capital. Am J Sociol 1988;94:S95-S120.

20. Beckles GL, Chou CF. Diabetes_United States, 2006 and 2010. MMWR Surveill Summ. 2013;62(suppl 3):99-104. 